\title{
Posicionamiento en espacios interiores con Android, Bluetooth y RSSI
}

\section{(Indoors positioning with Android, Bluetooth and RSSI)}

\author{
Edwin Cabrera-Goyes ${ }^{1}$, Diego Ordóñez-Camacho
}

\begin{abstract}
Resumen:
La investigación presentada tuvo dos objetivos. En primer lugar determinar de manera experimental la relación existente entre RSSI y distancia. En segundo lugar, mediante el uso de técnicas de trilateración, establecer la posición del receptor dentro del entorno en el cual se efectuó la experimentación. Las contribuciones principales son: determinar cómo los dispositivos Bluetooth interactúan entre ellos y las diferencias existentes al utilizar mediciones RSSI; recopilar data en distancias controladas y someter esta información a un análisis de regresión para establecer la relación entre RSSI y distancia; evaluar técnicas de trilateración de base para generar un prototipo funcional de posicionamiento en interiores con dispositivos Android. Los resultados son alentadores puesto que, inclusive con una configuración sumamente heterogénea de hardware y software y sin emplear ningún tipo de dispositivo especializado, fue posible obtener una alta precisión promedio.
\end{abstract}

Palabras clave: IPS; Bluetooth; RSSI; ubicación en interiores.

\begin{abstract}
:
This research has two main objectives. First, to determine experimentally the relationship between RSSI and distance. Second, with trilateration techniques, to establish the location of the receiver device in the experimental environment. The main contributions are: to determine how Bluetooth devices interact between them and how they differ when dealing with RSSI measures; to collect data over controlled distances applying to it regression analysis to establish the RSSI - distance relationship; to evaluate the basic trilateration techniques to produce a functional prototype for an indoor positioning system using Android devices. Results are encouraging considering that even with an extremely heterogeneous hardware and software configuration, it was possible to get a high average precision.
\end{abstract}

Keywords: IPS; Bluetooth; RSSI; indoors positioning.

\section{Introducción}

De manera general, en la actualidad la forma más difundida para ubicarse en el terreno es mediante el uso de algún tipo de Global Navigation Satellite System (GNSS). De entre estos sistemas, probablemente el más utilizado es el Global Positioning System (GPS) (Moser, Barišić, Rajle, \& Dimter, 2016), para el cual prácticamente todo teléfono inteligente cuenta con receptores. Sin embargo de su gran uso, la señal de los GNSS deja de ser útil en interiores o bajo techo; en aeropuertos, museos, centros comerciales y en edificios en general, es muy difícil y en muchos casos imposible obtener una ubicación precisa (Lu, Chen, Shen, Lam, \& Liu, 2007).

La problemática relacionada con la ubicación en interiores es actualmente uno de los campos de interés científico con mayor crecimiento y actividad. La investigación en los llamados Indoor Positioning Systems (IPS) (Mainetti, Patrono, \& Sergi, 2014), intenta brindar soluciones a casos de gran relevancia para mejorar la calidad de vida de, por ejemplo, personas con discapacidad visual. Para estos grupos, encontrar rápidamente y

\footnotetext{
1 Universidad Tecnológica Equinoccial, Quito - Ecuador ( \{cgep85623, dordonez\}@ute.edu.ec).
} 
de manera autónoma, por ejemplo, la ubicación de un cierto almacén en un centro comercial, o de una sala de clase en un centro de educación, puede marcar una gran diferencia en su forma de enfrentar el mundo, brindándoles un sentimiento ampliado de libertad en sus desplazamientos.

La oferta tecnológica en cuanto a sistemas de posicionamiento en interiores puede considerarse, aunque incipiente, variada. Existen sistemas experimentales sofisticados que, sin embargo, exigen que los dispositivos móviles-portátiles de posicionamiento cuenten con una variedad de sensores tales como brújula o magnetómetro (Tóth \& Tamás, 2016). No todos los dispositivos móviles (teléfonos inteligentes) de gama media-baja, disponibles en el mercado están equipados con esta clase de sensores.

En el mercado existen también otras alternativas en cuanto a dispositivos que pueden usarse para ubicación en interiores. Estas alternativas son aquellas que incluyen el componente de comunicación Bluetooth. En lo referente al presente estudio, estas alternativas son mucho más prácticas al incluirse en la mayoría de dispositivos móviles de gama media-baja disponibles en el mercado; no solo esto sino que este componente se encuentra con gran frecuencia en computadoras e inclusive en electrodomésticos (Asadullah \& Ullah, 2017). Todo esto facilita el uso de dispositivos con Bluetooth y al mismo tiempo reduce los costos dado que no es imprescindible usar equipamiento dedicado y especializado para los experimentos y se puede recurrir a productos estándar, asequibles en el mercado típico de consumidores.

Este trabajo, entonces, da seguimiento muy de cerca a estudios previos en el área (Cabrera-Goyes \& Ordóñez-Camacho, 2017) y se enfoca en la elaboración de un prototipo experimental de bajo presupuesto, para un sistema IPS. El prototipo utiliza como hardware principalmente dispositivos móviles Android estándar. Para utilizar dichos dispositivos se desarrolló un grupo de aplicaciones específicas las cuales explotan las capacidades del componente Bluetooth, más específicamente mediante el uso del Received Signal Strength Indication (RSSI) (Wu, Lee, Tseng, Jan, \& Chuang, 2008).

Los dispositivos se dividen en primer lugar entre emisores y receptores, sin ningún criterio en particular para este efecto. Los emisores son ubicados en lugares predeterminados cuya posición es minuciosamente registrada; al ejecutarse la aplicación del emisor, los dispositivos inician la emisión constante de la señal RSSI. Esta señal es capturada por los dispositivos receptores, los cuales usan su valor de intensidad para intentar calcular su posición en el entorno.

La investigación se dividió en dos fases; en la primera, se determinó de manera experimental la relación existente entre RSSI y distancia. La siguiente fase hizo uso de técnicas de trilateración para establecer la posición del receptor dentro del entorno en el cual se efectuó la experimentación. Los resultados son alentadores puesto que, inclusive con una configuración de hardware y software sumamente heterogénea y sin emplear ningún tipo de dispositivo especializado, fue posible obtener una precisión promedio de $84 \%$ (4.5 m en distancias de hasta $27 \mathrm{~m}$ ).

\subsection{Trabajos relacionados}

De manera amplia este trabajo se inscribe en el área de la Internet de las cosas, con especial énfasis en el uso de sensores inalámbricos (Alvear-Puertas, Rosero-Montalvo, Peluffo-Ordóñez, \& Pijal-Rojas, 2017; Quiñones-Cuenca, González-Jaramillo, Torres, \& Jumbo, 2017; Rojas, Bustos, \& Ordóñez-Camacho, 2017).

Más específicamente en el campo de los IPS, Xiao et al. (2016) exploran y reportan en la gran variedad de técnicas que se desarrollan actualmente. La principal división que conciben tiene que ver con el uso o no de dispositivos de ubicación por parte de los usuarios. Hay una variedad de técnicas en las cuales los usuarios no requerirían portar ningún tipo de dispositivo; es la infraestructura del entorno la encargada de detectar y reportar la posición del usuario. El costo de instalación en este tipo de sistemas puede ser alto. La otra división requiere que el usuario porte un dispositivo capaz de interactuar con 
la infraestructura para de esta manera establecer su posición. En estos casos los costos suelen ser menores dado que la infraestructura es mucho más ligera. En esta línea es frecuente el uso de radiofrecuencia y se encuentran investigaciones que exploran WiFi (Suárez et al., 2014), ZigBee (Larranaga, Muguira, López-Garde, \& Vázquez, 2010), RFID (Krukowski \& Arsenijevic, 2010) y Bluetooth (Torstensson, 2016) entre otras. Todas las técnicas presentan ventajas y desventajas (Mainetti et al., 2014) que, según el entorno y las necesidades específicas del proyecto, pueden resultar de utilidad.

Otro campo activo en el área está relacionado con la precisión en las mediciones y en las detecciones de posición. Feldmann, Kyamakya, Zapater, \& Lue (2003) hacen uso de receptores especializados BT-DG02 los cuales capturan la señal RSSI; reportan obtener una precisión del $74 \%$ con un error de $2.06 \mathrm{~m}$ dentro de un rango de $8 \mathrm{~m}$. Al hacer uso de dispositivos ZigBee, tanto a nivel de la emisión como de la recepción de la señal, Larranaga, Muguira, López-Garde, \& Vázquez (2010) experimentan en corredores de tamaño no especificado, y reportan que con el uso de 8 nodos emisores, obtienen un error de $4.24 \mathrm{~m}$. Cinefra (2014) utiliza solo 3 nodos emisores y reporta haber conseguido una precisión del $84 \%$ con un error de $1.5 \mathrm{~m}$ en distancias de hasta $9.6 \mathrm{~m}$. Mediante el uso de un sistema servidor centralizado, los dispositivos cliente comparten su posición, Zaidi et al. (2016) reportan una precisión de alrededor de $5 \mathrm{~m}$.

Existen también trabajos enfocados en la comparación de diversas técnicas de posicionamiento. Es así que Dahlgren \& Mahmood (2014) comparan dispositivos Bluetooth 2 y Bluetooth Smart para establecer sobre todo las diferencias de precisión al registrar la posición; encuentran que de manera general el hardware especializado es superior o más exacto a la hora de definir su ubicación en el entorno. Torstensson (2016) también profundiza en este tema y mediante el uso de faros Bluetooth Low Energy se concentra en el estudio de técnicas de reducción de ruido en la toma de medidas RSSI.

\subsection{Contribuciones}

Hay una variedad de alternativas de acción en el área de los IPS. En el caso de nuestro estudio, las contribuciones específicas en las cuales se centra son las siguientes:

- Determinar la manera en la cual los dispositivos Bluetooth interactúan entre ellos y las diferencias existentes al utilizar mediciones RSSI.

- Recopilar experimentalmente data sobre distancias controladas y someter esta información a análisis de regresión para encontrar la relación entre RSSI y distancia.

- Evaluar prácticamente las técnicas de trilateración de base para, en conjunto con las distintas aplicaciones construidas, generar un prototipo funcional de posicionamiento en interiores con dispositivos Android.

A continuación, en la Sección 2 se detallan los métodos, tecnologías y herramientas utilizadas; en la Sección 3 se presenta el sistema resultante, su funcionalidad, diseño y pruebas; finalmente, la Sección 4 presenta las principales conclusiones y recomendaciones de trabajo a futuro.

\section{Metodología}

Dos fases tuvieron lugar para la ejecución de este estudio; en primer lugar, una experimental en la cual se concibieron los prototipos de base para efectuar las mediciones en varios entornos y con distintas combinaciones de dispositivos; en segundo lugar, se desarrollaron las aplicaciones y pruebas finales.

Para determinar cómo los dispositivos Bluetooth interactúan entre ellos y la relación que existe entre el RSSI y la distancia, se realizó el primer prototipo de aplicación para la toma de datos; la aplicación se instaló en los dispositivos receptores. Se plantearon cuatro 
experimentos que midieron RSSI sobre distancias controladas y con línea de vista directa entre emisor y receptor; en la Tabla 1 se puede apreciar un resumen de estas experimentaciones cuya principal característica fue el cambio controlado de distancias con iteraciones de varias mediciones RSSI en cada punto. Los datos recopilados se analizaron en Weka (Witten, Frank, Hall, \& Pal, 2016) aplicando regresión lineal con validación cruzada de diez iteraciones.

Tabla 1. Experimentos efectuados, RSSI versus distancia

\begin{tabular}{lcccc}
\hline Experimento & $\mathbf{1}$ & $\mathbf{2}$ & $\mathbf{3}$ & $\mathbf{4}$ \\
\hline Rango & $0.6 \mathrm{~m}$ & $0-1 \mathrm{~m}$ & $1-5 \mathrm{~m}$ & $1-27 \mathrm{~m}$ \\
Cambio de posición & $\mathrm{N} / \mathrm{A}$ & $0.1 \mathrm{~m}$ & $0.5 \mathrm{~m}$ & $1 \mathrm{~m}$ \\
Iteraciones & 1 & 10 & 20 & 20 \\
Emisores & Ace & Ace, Z2 & Ace, S3, Htc & S3, 500 \\
Receptores & $\mathrm{X}$ & $\mathrm{X}$ & $\mathrm{X}, \mathrm{G} 3, \mathrm{~N} 3$ & $\mathrm{X}, \mathrm{N} 3$
\end{tabular}

Ace) Galaxy Ace; Z2) Xperia Z2; S3) Galaxy S3 mini; Htc) HTC Wildfire; X) Xperia X Perf; G3) LG-G3; 500) LG-p500h; N3) Galaxy Note 3

Luego, se analizaron las técnicas de posicionamiento y las variables y constantes sobre las cuales se tiene control para determinar el mejor método de posicionamiento. Posteriormente se prosiguió con el desarrollo del prototipo final de la aplicación sobre la base del prototipo utilizado en la fase experimental.

\section{Resultados y discusión}

A continuación, se analizan los resultados obtenidos, se presenta el sistema resultante, su funcionalidad, diseño y pruebas; se intercala, en cada ítem, una discusión comparativa con los trabajos relacionados.

\subsection{Experimentación}

Desde la primera experimentación se encontró que el RSSI presenta una gran variabilidad para una misma posición, como puede apreciarse en la Figura 1, donde luego de 10 mediciones los valores atípicos que determinan el rango son -68 y -55 , como se aprecia en la serie "Directo"; en la serie "Promedio-5" puede verse el efecto de tomar un promedio de las últimas 5 mediciones, donde adicionalmente observamos que con la quinta medición se alcanza ya el promedio total de -0.6. Una sola medición, luego, es insuficiente para obtener una distancia precisa. En la experimentación 2 se modificó la aplicación para realizar más de una iteración de toma de datos y promediarlos (Suárez et al., 2014). La misma idea fue aplicada en la investigación realizada por Torstensson (2016) en donde se promediaron 3 tomas de medidas. Se encontró que 5 valores RSSI permitían obtener un promedio razonablemente estable.

EI RSSI no se encuentra estandarizado; la escala de valores depende tanto del emisor como del receptor, es decir, dos receptores diferentes tendrán una escala diferente, aunque se trate del mismo emisor (Chowdhury et al., 2015).

La experimentación 2 consistió en 10 mediciones, con un mismo emisor y dos receptores diferentes alejándose $10 \mathrm{~cm}$ cada vez. En la Figura 2 puede observarse la diferencia del RSSI entre el Ace y el Z2; en promedio el Z2 detectó $12.9 \mathrm{dBm}$ menos que el Ace, con diferencias entre 0.3 y $19.4 \mathrm{dBm}$.

Estos datos permiten suponer que un proceso de calibración para cada dispositivo receptor podría solventar esta limitación, de esta manera sería posible establecer una 
relación más precisa entre RSSI y distancia, para un dispositivo específico. No se puede, sin embargo, dejar de lado la necesidad de contar con parámetros genéricos para cuando la calibración no es posible.

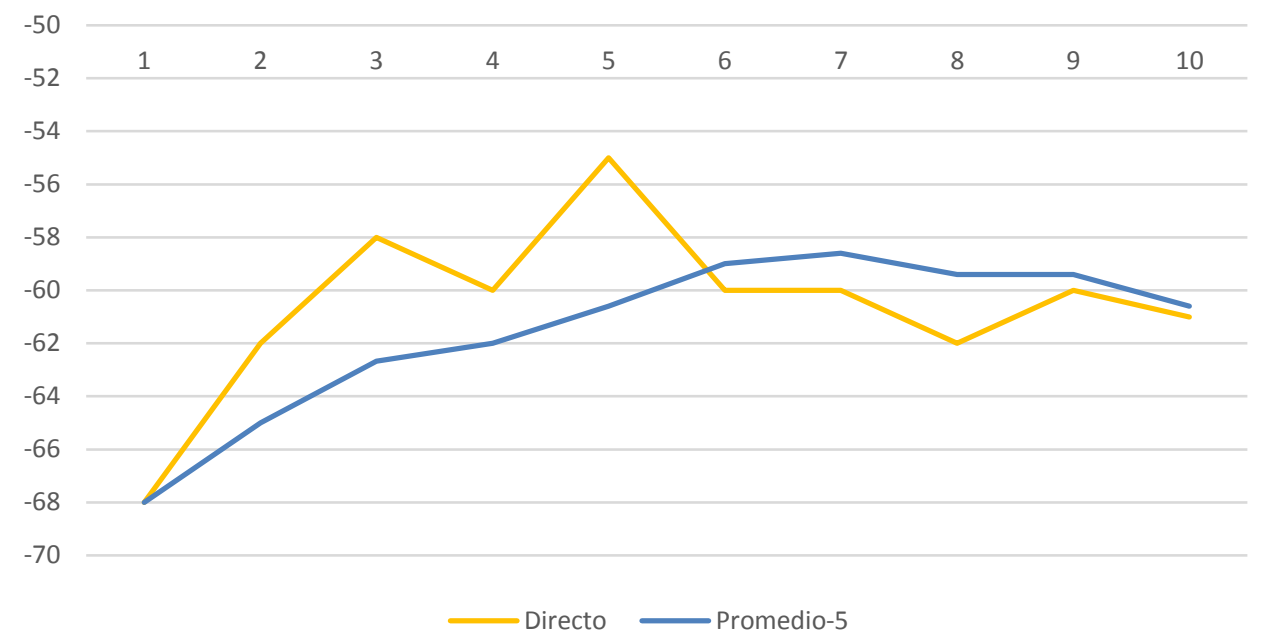

Figura 1. RSSI recolectados por el Sony Xperia X Performance a $0.6 \mathrm{~m}$ del Samsung Galaxy Ace

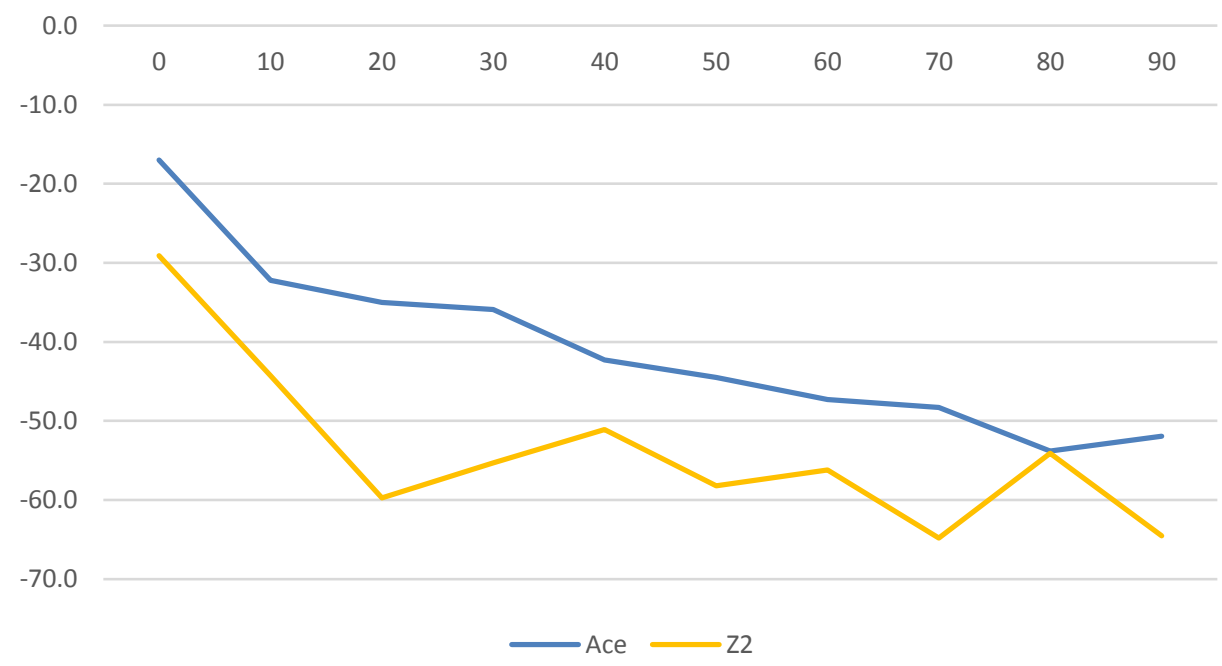

Figura 2. Comparación del promedio de RSSI de dos receptores diferentes respecto a un emisor común en diferentes distancias

La experimentación 3 se utilizó para probar la calibración de los dispositivos, al variar tanto emisores como receptores, en distancias entre $1-5 \mathrm{~m}$, con incrementos de $0.5 \mathrm{~m}$.

En la Figura 3, se presenta a manera de ejemplo la calibración de un dispositivo G3 al recibir datos de un Ace (las gráficas de calibración son similares para diferentes combinaciones de emisor-receptor); puede observarse que las mediciones RSSI directas presentan una gran variabilidad (línea RAW), la cual es suavizada tomando el promedio de las últimas 5 mediciones (línea Praw), con lo cual se logra un mejor ajuste a la tendencia de reducción de la señal según el incremento de la distancia (Linear).

Finalmente, en la experimentación 4 se hicieron pruebas con incremento del rango de distancia entre emisores y receptores entre 1 a $27 \mathrm{~m}$, con intervalos de $1 \mathrm{~m}$. Es interesante observar que el alcance dependerá mucho de las capacidades del hardware utilizado; Feldmann (2003), por ejemplo, encontró un límite de $8 \mathrm{~m}$ en el alcance utilizando 
dispositivos BT-DG02 con Bluetooth 1.1 clase 2, que teóricamente tienen un alcance de entre $10-20 \mathrm{~m}$. En el caso de esta experimentación el límite de $27 \mathrm{~m}$ se dio por el tamaño del espacio físico empleado más que por pérdida de la señal. Los datos recopilados se presentan en la Figura 4, donde puede observarse el gráfico de dispersión acumulado de las 4 tomas de datos.

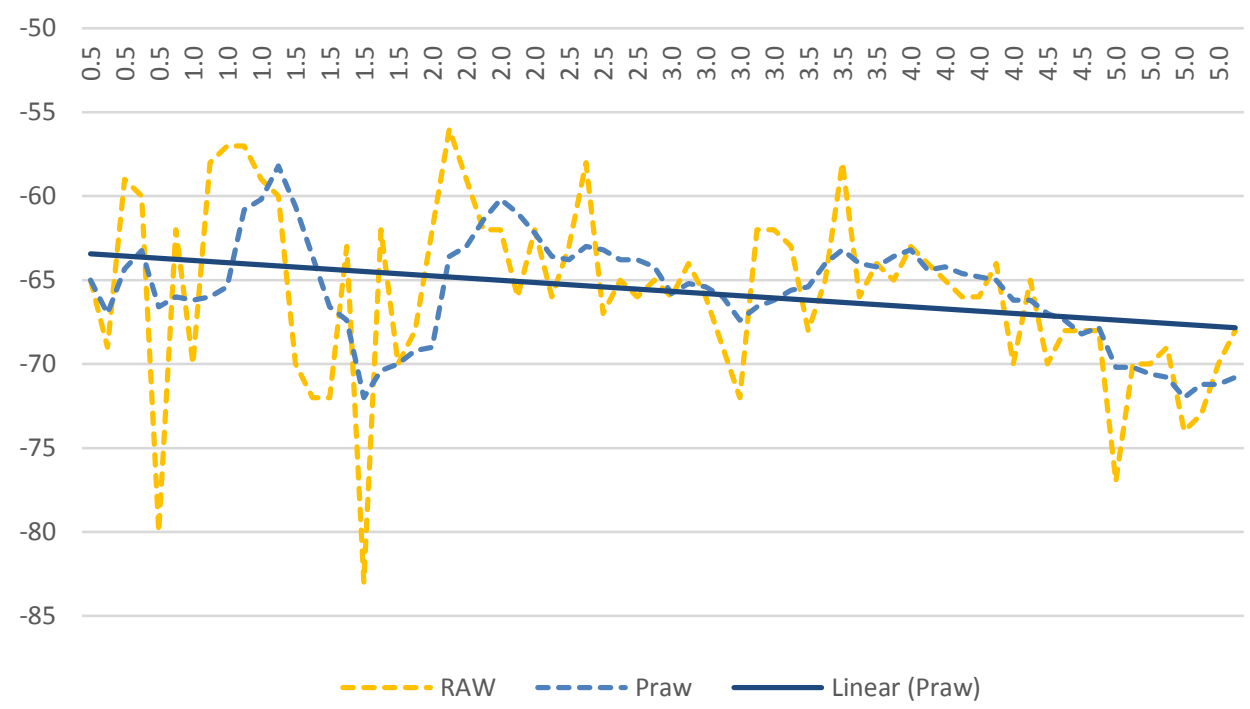

Figura 3. Calibración de un receptor LG G3, con un emisor Samsung Galaxy Ace

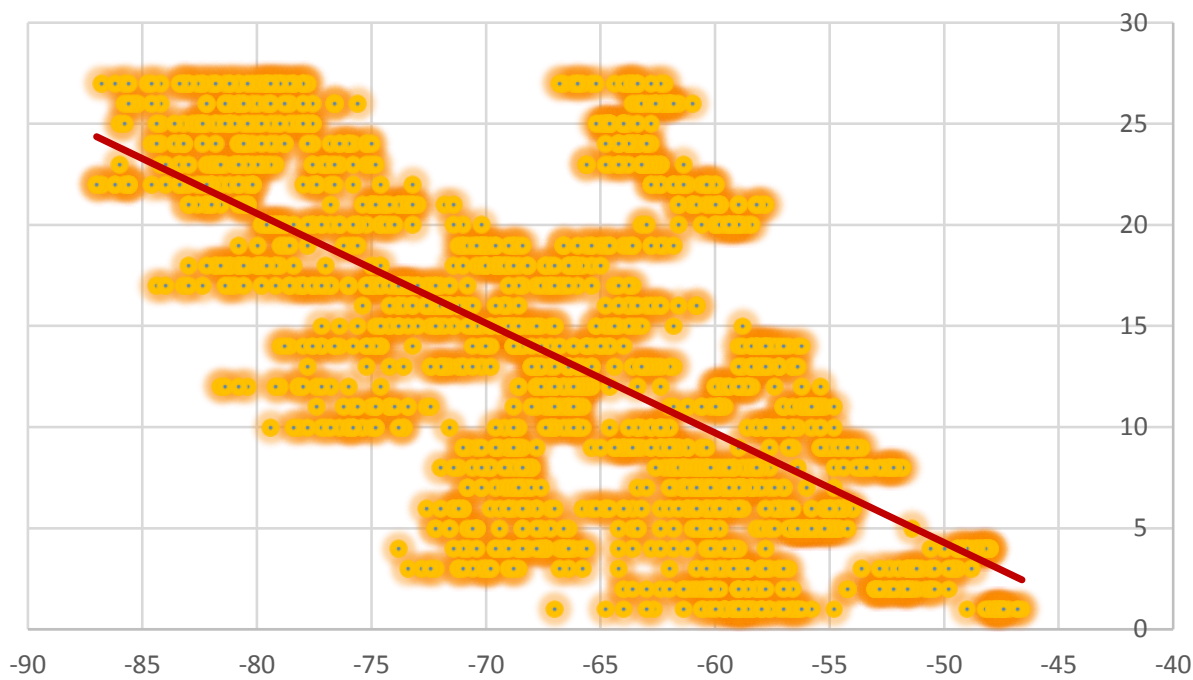

Figura 4. Dispersión global RSSI versus distancia

De esta data, mediante regresión lineal con validación cruzada de 10 iteraciones en Weka, se obtuvo la ecuación genérica para dispositivos no calibrados (1), donde $h_{d}$ es la distancia calculada y res el RSSI promedio de las últimas 5 tomas; el error global promedio de la medición es de $4.5 \mathrm{~m}$.

$$
h_{d}=-0.5425 r-22.838
$$

Cabe destacar, sin embargo, que ciertos dispositivos pueden presentar una precisión más alta y llegar el error en esta experimentación a ser de apenas $2.5 \mathrm{~m}$, como se ve en la Tabla 2, donde también se observa que el error global se ve fuertemente afectado por una sola medición extrema (en rojo). 
Tabla 2. Errores promedio en distancia calculada entre dispositivos emisores y receptores

\begin{tabular}{lll}
\hline & N3 & X \\
\hline 500 & 2.5 & 4.7 \\
S3 & 2.7 & 2.5 \\
& 3.6 & \\
& & 4.8 \\
\hline
\end{tabular}

Los resultados obtenidos son muy prometedores si se considera que se hace uso exclusivamente de dispositivos móviles estándar con Bluetooth. Otros estudios, como el de Cinefra (2014), proclaman errores menores, sin embargo, trabajan en distancias de hasta $9.6 \mathrm{~m}$ solamente y hacen uso de hardware especializado como faros Bluetooth Low Energy. Dahlgren \& Mahmood (2014) mencionan también que con hardware especializado como Bluetooth Smart se pueden obtener mejores resultados y conseguir un menor tiempo en el proceso de descubrimiento y por lo tanto mayor velocidad al momento de obtener la posición; también mencionan las posibilidades de mejora al incluir en el IPS otros dispositivos como acelerómetro y giroscopio.

\subsection{Implementación}

El prototipo, que se presenta en la Figura 5, permite registrar 3 dispositivos emisores para aplicar el método de trilateración (An, Xie, \& Ouyang, 2017; Rusli, Ali, Jamil, \& Din, 2016). Luego, en el modo de calibración (sección izquierda de la imagen), se pueden seleccionar distintas combinaciones de emisor y distancia para recolectar los datos que permitirán calcular la ecuación específica del dispositivo. Finalmente, en el modo de ubicación (sección derecha de la imagen), se inicia la recolección de datos, se calculan las distancias y se presenta gráficamente la ubicación estimada y el rango de error.

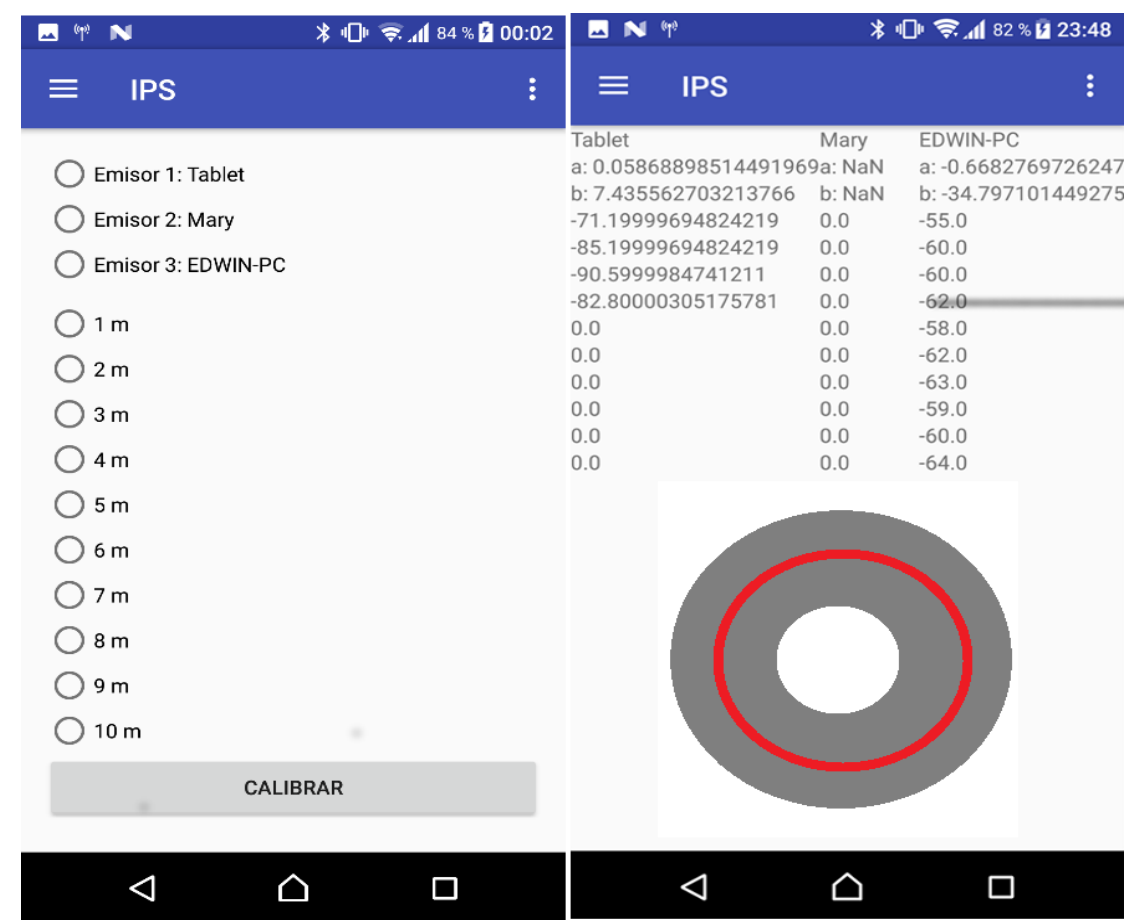

Figura 5. Pantallas de calibración y ubicación básica del prototipo 


\section{Conclusiones y recomendaciones}

Detectar la posición de un usuario a través del dispositivo que porta, se ha conseguido mediante el uso de Bluetooth y RSSI. Esto requiere en primer lugar determinar la distancia entre emisor y receptor, mediante la intensidad de la señal en $\mathrm{dBm}$, la cual disminuye según la distancia aumenta. Este cálculo ofrece al menos dos dificultades sustanciales que deben superarse: dispositivos diferentes pueden presentar variaciones de lecturas para una misma distancia, y la variación del RSSI fluctúa de manera indiscriminada en ciertas circunstancias. Estos problemas, si bien complejos, han podido ser minimizados en este estudio, en primer lugar al aplicar un suavizado de la señal mediante el promedio flotante de las tomas y en segundo lugar gracias a un proceso de calibración de los dispositivos receptores. Más aún, para aquellos casos en los cuales la calibración sería impráctica o imposible, se ha calculado una ecuación genérica para determinar la distancia a partir de la intensidad de la señal RSSI. Esta ecuación proporcionó una precisión destacable en el entorno experimental.

Este estudio tuvo como uno de sus objetivos utilizar hardware de consumidor o estándar. Ningún dispositivo especializado para IPS fue incluido en la experimentación. Pese a la limitación de este tipo de dispositivos, destacada ya en la literatura, gracias a las técnicas y aplicaciones de software empleadas, se obtuvieron resultados prometedores. Los niveles de precisión en el cálculo de la posición compiten con otros estudios que utilizan hardware especializado; inclusive, si se filtran mediciones extremas que muy posiblemente se debieron a problemas en los dispositivos, la precisión encontrada es superior que la de otros estudios.

El sistema muestra potencial y puede ser susceptible aun de muchas mejoras. Entre las principales y probablemente más urgentes consideramos que está la inclusión de filtros para tratar la señal RSSI con mayor precisión. Varias alternativas de filtros de ruido o suavizado deben ser integradas. De la misma manera, para el proceso de posicionamiento, se considera pertinente evaluar alternativas más avanzadas de triangulación y vincularlas con técnicas de ubicación predictiva.

\section{Bibliografía}

Alvear-Puertas, V., Rosero-Montalvo, P., Peluffo-Ordóñez, D., \& Pijal-Rojas, J. (2017). Internet de las Cosas y Visión Artificial, Funcionamiento y Aplicaciones: Revisión de Literatura. Enfoque UTE, 8(1), 244-256. https://doi.org/10.29019/enfoqueute.v8n1.121

An, K., Xie, S., \& Ouyang, Y. (2017). Reliable Sensor Location for Object Positioning and Surveillance via Trilateration. Transportation Research Procedia, 23, 228-245.

Asadullah, M., \& Ullah, K. (2017). Smart home automation system using Bluetooth technology. En Innovations in Electrical Engineering and Computational Technologies (ICIEECT), 2017 International Conference on (pp. 1-6). IEEE.

Cabrera-Goyes, E., \& Ordóñez-Camacho, D. (2017). Hacia un Sistema Bluetooth de Ubicación en Interiores con Dispositivos Estándar Android. En International Conference on Information Systems and Computer Science. IEEE Xplore (Por publicarse).

Chowdhury, T., Rahman, M., Parvez, S.-A., Alam, A., Basher, A., Alam, A., \& Rizwan, S. (2015). A multi-step approach for RSSI-based distance estimation using smartphones. En Networking Systems and Security (NSysS), 2015 International Conference on (pp. 1-5). IEEE.

Cinefra, N. (2014). An adaptive indoor positioning system based on Bluetooth Low Energy RSSI. Politecnico de Milano. Recuperado a partir de https://www.politesi.polimi.it/handle/10589/92284

Dahlgren, E., \& Mahmood, H. (2014). Evaluation of indoor positioning based on Bluetooth Smart technology. Chalmers University of Technology, Göteborg, Sweden. 
Feldmann, S., Kyamakya, K., Zapater, A., \& Lue, Z. (2003). An Indoor Bluetooth-Based Positioning System: Concept, Implementation and Experimental Evaluation. En International Conference on Wireless Networks (Vol. 272).

Krukowski, A., \& Arsenijevic, D. (2010). RFID-based positioning for building management systems. En Proceedings of 2010 IEEE International Symposium on Circuits and Systems (ISCAS) (pp. 3569-3572). https://doi.org/10.1109/ISCAS.2010.5537800

Larranaga, J., Muguira, L., López-Garde, J. M., \& Vázquez, J. I. (2010). An environment adaptive ZigBee-based indoor positioning algorithm. En 2010 International Conference on Indoor Positioning and Indoor Navigation (pp. 1-8). https://doi.org/10.1109/IPIN.2010.5647828

Lu, M., Chen, W., Shen, X., Lam, H.-C., \& Liu, J. (2007). Positioning and tracking construction vehicles in highly dense urban areas and building construction sites. Automation in Construction, 16(5), 647-656. https://doi.org/10.1016/j.autcon.2006.11.001

Mainetti, L., Patrono, L., \& Sergi, I. (2014). A survey on indoor positioning systems. En Software, Telecommunications and Computer Networks (SoftCOM), 2014 22nd International Conference on (pp. 111-120). IEEE.

Moser, V., Barišić, I., Rajle, D., \& Dimter, S. (2016). Comparison of different survey methods data accuracy for road design and construction. En Proceedings of the International Conference on Road and Rail Infrastructure CETRA.

Quiñones-Cuenca, M., González-Jaramillo, V., Torres, R., \& Jumbo, M. (2017). Sistema de Monitoreo de Variables Medioambientales usando una Red de Sensores Inalámbricos y Plataformas de Internet de las Cosas. Enfoque UTE, 8(1), 329-343. https://doi.org/10.29019/enfoqueute.v8n1.139

Rojas, J. P., Bustos, J. C., \& Ordóñez-Camacho, D. (2017). Transporte público inteligente al alcance de sus manos. Enfoque UTE, 8(1), 122-134. https://doi.org/10.29019/enfoqueute.v8n1.143

Rusli, M. E., Ali, M., Jamil, N., \& Din, M. M. (2016). An Improved Indoor Positioning Algorithm Based on RSSI-Trilateration Technique for Internet of Things (IOT). En Computer and Communication Engineering (ICCCE), 2016 International Conference on (pp. 72-77). IEEE.

Suárez, A., Santana, J. A., Macías-López, E. M., Mena, V. E., Canino, J. M., \& Marrero, D. (2014). RSSI prediction in WiFi considering realistic heterogeneous restrictions. Network Protocols and Algorithms, 6(4), 19-40.

Torstensson, D. (2016). Indoor Positioning System using Bluetooth Beacon Technology. Mälardalen University, Mälardalen, Sweden.

Tóth, Z., \& Tamás, J. (2016). Miskolc IIS hybrid IPS: Dataset for hybrid indoor positioning. En Radioelektronika (RADIOELEKTRONIKA), 2016 26th International Conference (pp. 408-412). IEEE.

Witten, I. H., Frank, E., Hall, M. A., \& Pal, C. J. (2016). Data Mining, Fourth Edition: Practical Machine Learning Tools and Techniques. Amsterdam Boston Heidelberg London New York Oxford Paris San Diego San Francisco Singapore Sydney Tokyo: Morgan Kaufmann.

Wu, R.-H., Lee, Y.-H., Tseng, H.-W., Jan, Y.-G., \& Chuang, M.-H. (2008). Study of characteristics of RSSI signal. En Industrial Technology, 2008. ICIT 2008. IEEE International Conference on (pp. 1-3). IEEE.

Xiao, J., Zhou, Z., Yi, Y., \& Ni, L. M. (2016). A Survey on Wireless Indoor Localization from the Device Perspective. ACM Comput. Surv., 49(2), 25:1-25:31. https://doi.org/10.1145/2933232

Zaidi, S., Assaf, A. E., Affes, S., \& Kandil, N. (2016). Accurate Range-Free Localization in Multi-Hop Wireless Sensor Networks. IEEE Transactions on Communications, 64(9), 3886-3900. https://doi.org/10.1109/TCOMM.2016.2590436 